\title{
MTBseq: A comprehensive pipeline for whole genome sequence analysis of Mycobacterium tuberculosis complex isolates
}

Thomas Andreas Kohl ${ }^{1}$, Christian Utpatel ${ }^{1}$, Viola Schleusener ${ }^{1}$, Maria Rosaria De Filippo ${ }^{2}$, Patrick Beckert 1,3 , Daniela Maria Cirillo ${ }^{2}$, Stefan Niemann ${ }^{\text {Corresp. }{ }^{1,3}}$

1 Molecular and Experimental Mycobacteriology, Research Center Borstel, Borstel, Germany

2 Emerging Bacterial Pathogens Unit, Division of Immunology, Transplantation and Infectious Diseases, IRCCS San Raffaele Scientific Institute, Milan, Italy

3 German Center for Infection Research (DZIF), partner site Hamburg - Lübeck - Borstel - Riems, Borstel, Deutschland

Corresponding Author: Stefan Niemann

Email address: sniemann@fz-borstel.de

Analyzing whole genome sequencing data of Mycobacterium tuberculosis complex isolates (MTBC) in a standardized workflow enables both comprehensive antibiotic resistance profiling and outbreak surveillance with highest resolution up to the identification of recent transmission chains. Here we present MTBseq, a bioinformatics pipeline for next generation genome sequence data analysis of MTBC isolates. Employing a reference mapping based workflow, MTBseq reports detected variant positions annotated with known association to antibiotic resistance and performs a lineage classification based on phylogenetic single nucleotide polymorphisms (SNPs). When comparing multiple datasets, MTBseq provides a joint list of variants and a FASTA alignment of SNP positions for use in phylogenomic analysis, and identifies groups of related isolates. The pipeline is customizable, expandable and can be used on a desktop computer or laptop without any internet connection, ensuring mobile usage and data security. MTBseq and accompanying documentation is available from https://github.com/ngs-fzb/MTBseq_source. 
1 MTBseq: A comprehensive pipeline for whole genome sequence analysis of Mycobacterium

2 tuberculosis complex isolates

3 Thomas Andreas Kohl11,\#, Christian Utpatel ${ }^{1, \#}$, Viola Schleusener ${ }^{1}$, Maria Rosaria De Filippo ${ }^{3}$,

4 Patrick Beckert ${ }^{1,2}$, Daniela Maria Cirillo ${ }^{3}$, Stefan Niemann ${ }^{1,2}$

5

$6{ }^{1}$ Molecular and Experimental Mycobacteriology, Research Center Borstel, Borstel, Germany ${ }^{2}$

7 German Center for Infection Research (DZIF), partner site Hamburg - Lübeck - Borstel - Riems,

8 Borstel, Germany

$9{ }^{3}$ Emerging Bacterial Pathogens Unit, Division of Immunology, Transplantation and Infectious

10 Diseases, IRCCS San Raffaele Scientific Institute, Milan, Italy

12 \# Contributed equally.

13

14

15

16

17

18

19

20

21

22

23

24

25 
Abstract: Analyzing whole genome sequencing data of Mycobacterium tuberculosis complex isolates (MTBC) in a standardized workflow enables both comprehensive antibiotic resistance profiling and outbreak surveillance with highest resolution up to the identification of recent transmission chains. Here we present MTBseq, a bioinformatics pipeline for next generation genome sequence data analysis of MTBC isolates. Employing a reference mapping based workflow, MTBseq reports detected variant positions annotated with known association to antibiotic resistance and performs a lineage classification based on phylogenetic single nucleotide polymorphisms (SNPs). When comparing multiple datasets, MTBseq provides a joint list of variants and a FASTA alignment of SNP positions for use in phylogenomic analysis, and identifies groups of related isolates. The pipeline is customizable, expandable and can be used on a desktop computer or laptop without any internet connection, ensuring mobile usage and data security.

MTBseq and accompanying documentation is available from $<$ https://github.com/ngsfzb/MTBseq_source>.

\section{Introduction}

53

The recent development of next generation sequencing (NGS) technologies in line with the reduction of sequencing costs and introduction of benchtop instruments allows the use of whole genome sequencing (WGS) as routine tool for bacterial strain characterization e.g. for resistance prediction and in-depth genotyping of bacterial isolates (Walker et al. 2017). This development has led to significant improvements for the epidemiological surveillance of major pathogens such as the Mycobacterium tuberculosis complex (MTBC), the causative agent of tuberculosis (TB) (Dheda et al., 2017; Merker et al., 2017; Walker et al., 2018; Zignol et al., 2018). With 10.4 million new cases in 2016 and the emergence of multidrug resistant strains, tuberculosis remains one of the ten leading causes of death worldwide (World Health Organization, 2017). 
The application of WGS technologies clearly advances resistance prediction, outbreak detection,

65

66

67

68

and genomic surveillance of MTBC (Merker et al., 2017). At the same time, no comprehensive pipeline allowing for the full analysis of individual datasets and a set of samples has been proposed so far. Comprehensive and powerful open source packages for standardized NGS analysis exist such as UGENE (Okonechnikov et al., 2012), The Galaxy Project (Afgan et al., 2016), or GenePattern (Reich et al., 2006), as well as programming language toolkits such as BioPerl (Stajich et al., 2002), BioPython (Cock et al., 2009), or BioRuby (Goto et al., 2010). However, to set up fully integrated workflows from scratch that allow for an accurate and meaningful analysis of NGS data from clinical MTBC strains, still requires programming expertise and trained bioinformatics personnel. This constrains the application of NGS analysis to specialized laboratories, leads to a huge diversity of analysis pipelines with group specific solutions and seriously complicates comparison of results. Several automated pipelines for resistance determination have been developed, namely three web services (CASTB [Iwai et al., 2015], PhyResSE [Feuerriegel et al., 2015], and TBProfiler [Coll et al., 2015]), and two local software solutions (KvarQ [Steiner et al., 2014] and Mykrobe Predictor TB [Bradley et al., 2015]). All these tools enable non-specialists to infer drug resistance from WGS data of MTBC strains and also provide phylogenetic classification results. Their respective strengths and weaknesses have been compared before (Schleusener et al., 2017).

Still, bioinformatics data analysis is a clear bottleneck that restricts accessibility and wide adoption of NGS technologies in TB research, diagnostics and surveillance.

To address this challenge, we developed MTBseq, an automated pipeline for MTBC NGS data analysis. MTBseq combines all necessary steps for the analysis of NGS datasets from MTBC strains ranging from basic analysis procedures such as mapping of reads to the reference genome, to detection of variant positions annotated with known association to antibiotic resistance, and lineage classification based on phylogenetic single nucleotide polymorphisms (SNPs). In addition, MTBseq enables the comparative analysis of multiple samples to produce joint lists of variants and a FASTA alignment of SNP positions for use with tree-based approaches. 
93

94

95

96

97

98

99

100

101

102

103

104

105

106

107

108

109

110

111

112

113

114

115

116

117

118

119

120

121

122

123

\section{Material \& Methods}

\section{Description of MTBseq individual steps}

MTBseq employs the widely used open source programs BWA (Li, 2009), SAMtools (Li et al., 2009), PICARD-tools (https://broadinstitute.github.io/picard/) and Genome Analysis Toolkit (GATK) (McKenna et al., 2010). The workflow starts with raw FASTQ formatted sequences (reads). Within the mapping step, the BWA-MEM algorithm and SAMtools are used for a generalized mapping procedure (TBbwa). The output is a deduplicated and sorted alignment file saved in the binary alignment format BAM (Li et al., 2009). Next, GATK is used for base call recalibration and realignment of reads around insertions or deletions (TBrefine). Variant calling is a multi-step process that starts with the SAMtools mpileup utility, providing coverage information at single base resolution (TBpile) and employs thresholds for coverage and base quality (TBlist). With default settings, variants need to be indicated by four reads mapped in each forward and reverse orientation, respectively, at 75\% allele frequency, and by at least 4 calls with a phred score of at least 20. Therefore, MTBseq will report reliably detected variants if they are present in about $75 \%$ or more of the bacterial population when using default values. In the sample specific report file, detected SNPs and insertions or deletions are annotated with respective metadata, including resulting amino acid changes for SNPs in coding regions and association to antibiotic resistance (TBvariants). The sixth step creates a descriptive statistics report of the mapping and variant detection steps, giving a clear indication of overall dataset performance (TBstats). The last sample specific module enables the phylogenetic classification of the input sample(s) according to phylogenetically informative SNPs from the literature (Coll et al., 2014; Homolka et al., 2012; Merker et al. 2015) (TBstrains). A comparative analysis of multiple samples is provided by the TBjoin and TBamend modules, which can be executed for any set of samples already processed by the sample specific workflow. The primary result is a list, containing information of all positions for which a variant had been detected in any of the input samples. To facilitate phylogenetic analysis, variant subsets are automatically generated, filtered for repetitive regions (Comas et al., 2010) and resistance-associated genes, the kind of variant detected, and the presence of other variants within a window of $12 \mathrm{bp}$ within the same dataset (Walker et al., 2013). In addition, FASTA formatted sequences are generated as direct input for targeted applications (e.g. tree reconstruction algorithms). The comparative analysis 
124 finishes with grouping input samples according to the number of distinct SNP positions

125 (TBgroups).

126

127

Although MTBseq offers a batch mode, the described modules are functionally separated and independent in execution (Fig. 1, blue boxes). This architecture allows every single module to be executed directly by the user and ensures expandability of the workflow by developers. A simple checkpoint system is implemented between every step (Fig. 1, blue lines with diamond symbol), keeping track of analysis results already generated. Therefore, the pipeline parts are executed only for samples that have the respective input and lack the respective output of the module invoked. In order to ensure this functionality, MTBseq creates its own working environment at the location of execution. With MTBseq, we aim to provide a sequence analysis pipeline for the MTBC that is customizable, expandable, user friendly and standardized. At the same time, we aim to offer users the opportunity to customize the functionality to their specific needs. Therefore, all parameters are set to default values while especially thresholds within variant calling and in the comparative part of the program can be easily modified by the user. In this way, we want to enable any user with basic Linux experience to run the software, while also allowing for the easy modification of the functionality by users with a bioinformatics background.

MTBseq uses the M. tuberculosis H37Rv genome (NC_000962.3) and corresponding metadata as reference by default but the pipeline can be used with any MTBC or non-MTBC bacterial reference genome and corresponding annotation, supplied by the user. For base quality recalibration and annotation of resistance associated or phylogenetic variants, a default list is provided with MTBseq, which can be easily replaced by the user with a respective compilation, e.g. as drawn from the ReSeqTB or CRyPTIC initiatives. This is especially important since this data forms the basis for inferring a resistance profile from the detected variants.

\section{Programming language and availability}

152 MTBseq is written in the Perl programming language and can be obtained from

$153<$ https://github.com/ngs-fzb/MTBseq_source>, including the full source code, documentation, 154 and usage guidelines. 
156

157

158

159

160

161

162

163

164

165

166

167

168

169

170

171

172

173

174

175

176

177

178

179

180

181

182

183

184

\section{Results}

\section{General workflow of MTBseq}

MTBseq consists of two workflows with seven modules encompassing a sample specific analysis and three modules enabling comparative analysis of multiple datasets (Fig. 1). The sample specific workflow comprises read alignment to the M. tuberculosis $\mathrm{H} 37 \mathrm{Rv}$ reference genome (NC_000962.3), refining the resulting alignment, and the detection of single nucleotide polymorphisms (SNPs), as well as insertions and deletions. Reported variants are annotated with applicable metadata, in particular whether an association with antibiotics resistance is known. In addition, MTBseq performs a phylogenetic classification using a set of informative SNPs (Coll et al., 2014; Homolka et al., 2012; Merker et al. 2015).

For multiple datasets, the pipeline enables a comparative analysis of the analysis results of a set of samples (Fig. 1), which includes an agglomerative cluster analysis (e.g. inference of transmission groups from pairwise distances) and the determination of informative positions for the reconstruction of phylogenetic trees. MTBseq can be executed in a batch mode without any user intervention. This is an important issue, if large numbers of datasets have to be analyzed. Details of individual steps are detailed in the Materials and Methods section. Importantly, the pipeline can be run nearly completely automated from one command line call, with all parameters pre-set to appropriate default values.

\section{Antibiotic resistance detection and classification}

Using procedures similar to a recently published study (Schleusener et al., 2017), we performed a systematic evaluation of MTBseq for the prediction of antibiotic resistance against the four first line drugs in tuberculosis therapy (isoniazid, rifampicin, ethambutol, and pyrazinamide) and streptomycin, as well as for phylogenetic classification of MTBC isolates. The dataset used consisted of 91 well-characterized strains of a collection from Sierra Leone, for which both WGS (ENA accession number PRJEB7727) and Sanger sequencing data was available (Schleusener et al., 2017). We compared the results with five other software solutions available for resistance inference and phylogenetic classification of MTBC datasets, i.e. CASTB (Iwai et al., 2015), KvarQ (Steiner et al., 2014), Mykrobe Predictor TB (Bradley et al., 2015), PhyResSE 
185 (Feuerriegel et al., 2015), and TBProfiler (Coll et al., 2015). Overall, MTBseq, PhyResSE, and

186 TBProfiler exhibited the highest sensitivity and specificity among the tools tested (Table 1).

187

188

189

190

191

192

193

194

195

196

197

198

199

200

201

202

203

204

205

206

207

208

209

210

211

212

213

214 215

Using MTBseq, we obtained a 100\% sensitivity compared to Sanger sequencing results for rifampicin and isoniazid, the most important drugs for TB treatment. For ethambutol, streptomycin, and pyrazinamide, the sensitivity was also $100 \%$, and specificity at least $90 \%$ (Table 1). In these calculations, we included the detection of insertions or deletions in genes annotated by MTBseq as resistance associated (Table S1) and the detection of resistant subpopulations, which MTBseq reported correctly when parameters were adjusted to detect low frequency variants (Table S1). In the low frequency detection mode (set with the "-lowfreq_vars" option), MTBseq will consider the majority allele different from the wild type base. Regarding the classification of samples into known phylogenetic lineages, MTBseq was able to classify 11 strains not resolved by classical genotyping (classical genotyping result: "none"; Table S1). As by MTBseq default settings, a set of known variant positions was used for base quality calibration (Table S2).

\section{Phylogenetic analysis and cluster detection}

We used a well characterized dataset of 26 isolates from an outbreak in Hamburg to perform a phylogenetic analysis with MTBseq (Kohl et al., 2014). It contains next generation sequencing data from 26 isolates from a longitudinal population-based study in Hamburg, which were recognized as belonging to a potential outbreak as all isolates presented with the same classical genotyping patterns. All 26 isolates were analyzed with MTBseq in a joint comparison using parameters set to default values, with 4,304,720 out of the 4,411,532 bp of the H37Rv reference genome fulfilling the thresholds for variant detection. In total, 988 SNP positions were identified for a phylogenomic analysis, and the FASTA file produced by MTBseq was used as input for the tree construction program FastTree 2 (Price et al., 2010).

In addition, MTBseq was configured to detect clustered isolates with a threshold of $12 \mathrm{bp}$ to the nearest cluster member. Both the constructed tree and the clusters indicate a central group of 22 isolates forming a tight cluster, and four isolates (1024-01, 3929-10, 6631-04, 6821-03) not related to this outbreak (Fig. 2). The same can be seen in the matrix of pairwise distances (Fig. 3). 


\section{Discussion}

218

We developed MTBseq to overcome constraints in the bioinformatics analysis of NGS data from clinical MTBC strains and to provide a standard analysis pipeline to increase the accessibility and adoption of NGS technologies in TB research, diagnostics and surveillance. MTBseq employs a reference mapping based workflow, reports detected variant positions annotated with known association to antibiotic resistance and performs a lineage classification based on phylogenetic single nucleotide polymorphisms (SNPs). In the joint analysis of multiple datasets, MTBseq provides a joint list of variants, a SNP distance matrix, a FASTA alignment of SNP positions for use in phylogenomics, and identifies groups of related isolates.

227

Here we demonstrated the sensitivity and accuracy for resistance profiling and genotyping of MTBseq. Compared to Sanger sequencing results we obtained a 100\% sensitivity for rifampicin, isoniazid, ethambutol, streptomycin, and pyrazinamide with the specificity being at least $90 \%$ for the latter four. For the correct detection of resistance-associated minority variants the low frequency detection mode of MTBseq had to be employed in which the majority allele other than wild type is considered per position. This mode should preferably be used for detection of resistance-associated variants if resistant subpopulations are suspected. This could be the case during early stages of drug-resistance acquisition or mixed TB infections. Here, it is important to keep in mind that due to the lack of an accessible gold standard sensitivity of specificity of the low frequency mode have not yet been evaluated. The phylogenetic classification into lineages and sublineages of the MTBC by MTBseq was in overall concordance with results from traditional genotyping and on par with PhyResSE and TBProfiler.

The results of the phylogenetic analysis and cluster detection of 26 isolates from an outbreak in Hamburg are in full agreement with published findings for this dataset (Kohl et al., 2014), which also identified a central cluster of 22 isolates and the same four outlying isolates. For tree construction, we chose the program FastTree 2, but as MTBseq provides a full FASTA alignment of SNP positions for phylogenetic analysis any phylogenomic suite can be used. 
247

248

249

250

251

252

253

254

255

256

257

258

259

260

261

262

263

264

265

266

267

268

269

270

271

272

273

274

275

276

277

278

279

280

281

\section{Conclusions}

MTBseq provides a comprehensive analysis pipeline for WGS analysis of MTBC NGS data. The pipeline is fully customizable and the functionality can be easily adjusted and extended, both by modifying the implementation and by adding further modules to the respective workflows. At the same time, the pipeline can be run nearly completely automated from one command line call, with all parameters pre-set to appropriate default values. We demonstrated the accuracy and sensitivity for resistance profiling, genotyping, and comparative analysis, concluding that MTBseq is a suitable automated solution for resistance deduction, and phylogenetic classification and analysis of MTBC whole genome datasets. The full source code with accompanying documentation and usage guidelines is provided at $<$ https://github.com/ngsfzb/MTBseq_source $>$. MTBseq thus provides a full automatized analysis pipeline for NGS datasets from MTBC strains, further paving the way for efficient application of WGS in the characterization of bacterial pathogens.

\section{Acknowledgements}

The authors thank Robin Koch, Alexandra Dangel, Conor Meehan, and Matthias Merker for their thorough testing of the MTBseq package and helpful input during development.

\section{References}

Afgan, E., Baker, D., van den Beek, M., Blankenberg, D., Bouvier, D., Cech, M., Chilton, J., Clements, D., Coraor, N., Eberhard, C., Gruning, B., Guerler, A., Hillman-Jackson, J., Von Kuster, G., Rasche, E., Soranzo, N., Turaga, N., Taylor, J., Nekrutenko, A. and Goecks, J. 2016. The Galaxy platform for accessible, reproducible and collaborative biomedical analyses: 2016 update. Nucleic Acids Research 44: W3-W10 DOI: $10.1093 /$ nar/gkw343.

Bradley, P., Gordon, N. C., Walker, T. M., Dunn, L., Heys, S., Huang, B., Earle, S., Pankhurst, L. J., Anson, L., de Cesare, M., Piazza, P., Votintseva, A. A., Golubchik, T., Wilson, D. J., Wyllie, D. H., Diel, R., Niemann, S., Feuerriegel, S., Kohl, T. A., Ismail, N., Omar, S. V., Smith, E. G., Buck, D., McVean, G., Walker, A. S., Peto, T. E., Crook, D. W. and Iqbal, Z. 2015. Rapid antibiotic-resistance predictions from genome sequence data for Staphylococcus aureus and Mycobacterium tuberculosis. Nature Communications 6: 10063 DOI: $10.1038 /$ ncomms10063.

Cock, P. J., Antao, T., Chang, J. T., Chapman, B. A., Cox, C. J., Dalke, A., Friedberg, I., Hamelryck, T., Kauff, F., Wilczynski, B. and de Hoon, M. J. 2009. Biopython: freely available Python tools for computational 
282

283

284

285

286

287

288

289

290

291

292

293

294

295

296

297

298

299

300

301

302

303

304

305

306

307

308

309

310

311

312

313

314

315

316

317

318

319

320

321

322

323

324

325

326

327

328

molecular biology and bioinformatics. Bioinformatics 25: 1422-1423 DOI:

10.1093/bioinformatics/btp163.

Coll, F., McNerney, R., Guerra-Assuncao, J. A., Glynn, J. R., Perdigao, J., Viveiros, M., Portugal, I., Pain, A., Martin, N. and Clark, T. G. 2014. A robust SNP barcode for typing Mycobacterium tuberculosis complex strains. Nature Communications 5: 4812 DOI: 10.1038/ncomms5812.

Coll, F., McNerney, R., Preston, M. D., Guerra-Assuncao, J. A., Warry, A., Hill-Cawthorne, G., Mallard, K., Nair, M., Miranda, A., Alves, A., Perdigao, J., Viveiros, M., Portugal, I., Hasan, Z., Hasan, R., Glynn, J. R., Martin, N., Pain, A. and Clark, T. G. 2015. Rapid determination of anti-tuberculosis drug resistance from whole-genome sequences. Genome Medicine 7: 51 DOI: 10.1186/s13073-015-0164-0.

Comas, I., Chakravartti, J., Small, P. M., Galagan, J., Niemann, S., Kremer, K., Ernst, J. D. and Gagneux, S. 2010. Human T cell epitopes of Mycobacterium tuberculosis are evolutionarily hyperconserved. Nature Genetics 42: 498-503 DOI: 10.1038/ng.590.

Dheda, K., Gumbo, T., Maartens, G., Dooley, K. E., McNerney, R., Murray, M., Furin, J., Nardell, E. A., London, L., Lessem, E., Theron, G., van Helden, P., Niemann, S., Merker, M., Dowdy, D., Van Rie, A., Siu, G. K., Pasipanodya, J. G., Rodrigues, C., Clark, T. G., Sirgel, F. A., Esmail, A., Lin, H. H., Atre, S. R., Schaaf, H. S., Chang, K. C., Lange, C., Nahid, P., Udwadia, Z. F., Horsburgh, C. R., Jr., Churchyard, G. J., Menzies, D., Hesseling, A. C., Nuermberger, E., Mcllleron, H., Fennelly, K. P., Goemaere, E., Jaramillo, E., Low, M., Jara, C. M., Padayatchi, N. and Warren, R. M. 2017. The epidemiology, pathogenesis, transmission, diagnosis, and management of multidrug-resistant, extensively drug-resistant, and incurable tuberculosis. The Lancet Respiratory Medicine DOI: 10.1016/S2213-2600(17)30079-6.

Feuerriegel, S., Schleusener, V., Beckert, P., Kohl, T. A., Miotto, P., Cirillo, D. M., Cabibbe, A. M., Niemann, S. and Fellenberg, K. 2015. PhyResSE: a Web Tool Delineating Mycobacterium tuberculosis Antibiotic Resistance and Lineage from Whole-Genome Sequencing Data. Journal of Clinical Microbiology 53: 1908-1914 DOI: 10.1128/JCM.00025-15.

Goto, N., Prins, P., Nakao, M., Bonnal, R., Aerts, J. and Katayama, T. 2010. BioRuby: bioinformatics software for the Ruby programming language. Bioinformatics 26: 2617-2619 DOI:

10.1093/bioinformatics/btq475.

He, Z., Zhang, H., Gao, S., Lercher, M. J., Chen, W. H. and Hu, S. 2016. Evolview v2: an online visualization and management tool for customized and annotated phylogenetic trees. Nucleic Acids Research 44: W236-241 DOI: 10.1093/nar/gkw370.

Homolka, S., Projahn, M., Feuerriegel, S., Ubben, T., Diel, R., Nubel, U. and Niemann, S. 2012. High resolution discrimination of clinical Mycobacterium tuberculosis complex strains based on single nucleotide polymorphisms. PLoS One 7: e39855 DOI: 10.1371/journal.pone.0039855.

Iwai, H., Kato-Miyazawa, M., Kirikae, T. and Miyoshi-Akiyama, T. 2015. CASTB (the comprehensive analysis server for the Mycobacterium tuberculosis complex): A publicly accessible web server for epidemiological analyses, drug-resistance prediction and phylogenetic comparison of clinical isolates. Tuberculosis (Edinburgh) 95: 843-844 DOI: 10.1016/j.tube.2015.09.002. 
354

355

356

357

358

359

360

361

362

363

364

365

366

367

368

369

370

371

372

373

374

375

376

Kohl, T. A., Diel, R., Harmsen, D., Rothganger, J., Walter, K. M., Merker, M., Weniger, T. and Niemann, S. 2014. Whole-genome-based Mycobacterium tuberculosis surveillance: a standardized, portable, and expandable approach. Journal of Clinical Microbiology 52: 2479-2486 DOI: 10.1128/JCM.00567-14.

Li, H. and Durbin, R. 2009. Fast and accurate short read alignment with Burrows-Wheeler transform. Bioinformatics 25: 1754-1760 DOI: 10.1093/bioinformatics/btp324.

Li, H., Handsaker, B., Wysoker, A., Fennell, T., Ruan, J., Homer, N., Marth, G., Abecasis, G., Durbin, R. and Genome Project Data Processing, S. 2009. The Sequence Alignment/Map format and SAMtools.

Bioinformatics 25: 2078-2079 DOI: 10.1093/bioinformatics/btp352.

McKenna, A., Hanna, M., Banks, E., Sivachenko, A., Cibulskis, K., Kernytsky, A., Garimella, K., Altshuler, D., Gabriel, S., Daly, M. and DePristo, M. A. 2010. The Genome Analysis Toolkit: a MapReduce framework for analyzing next-generation DNA sequencing data. Genome Research 20: 1297-1303 DOI: 10.1101/gr.107524.110.

Merker, M., Blin, C., Mona, S., Duforet-Frebourg, N., Lecher, S., Willery, E., Blum, M. G., Rusch-Gerdes, S., Mokrousov, I., Aleksic, E., Allix-Beguec, C., Antierens, A., Augustynowicz-Kopec, E., Ballif, M., Barletta, F., Beck, H. P., Barry, C. E., 3rd, Bonnet, M., Borroni, E., Campos-Herrero, I., Cirillo, D., Cox, H., Crowe, S., Crudu, V., Diel, R., Drobniewski, F., Fauville-Dufaux, M., Gagneux, S., Ghebremichael, S., Hanekom, M., Hoffner, S., Jiao, W. W., Kalon, S., Kohl, T. A., Kontsevaya, I., Lillebaek, T., Maeda, S., Nikolayevskyy, V., Rasmussen, M., Rastogi, N., Samper, S., Sanchez-Padilla, E., Savic, B., Shamputa, I. C., Shen, A., Sng, L. H., Stakenas, P., Toit, K., Varaine, F., Vukovic, D., Wahl, C., Warren, R., Supply, P., Niemann, S. and Wirth, T. 2015. Evolutionary history and global spread of the Mycobacterium tuberculosis Beijing lineage. Nature Genetics 47: 242-249 DOI: 10.1038/ng.3195.

Merker, M., Kohl, T. A., Niemann, S. and Supply, P. 2017. The Evolution of Strain Typing in the Mycobacterium tuberculosis Complex. Advances in Experimental Medicine and Biology 1019: 43-78 DOI: 10.1007/978-3-319-64371-7_3.

Okonechnikov, K., Golosova, O., Fursov, M. and team, U. 2012. Unipro UGENE: a unified bioinformatics toolkit. Bioinformatics 28: 1166-1167 DOI: 10.1093/bioinformatics/bts091.

Price, M. N., Dehal, P. S. and Arkin, A. P. 2010. FastTree 2--approximately maximum-likelihood trees for large alignments. PLoS One 5: e9490 DOI: 10.1371/journal.pone.0009490.

Reich, M., Liefeld, T., Gould, J., Lerner, J., Tamayo, P. and Mesirov, J. P. 2006. GenePattern 2.0. Nature Genetics 38: 500-501 DOI: 10.1038/ng0506-500.

Schleusener, V., Koser, C. U., Beckert, P., Niemann, S. and Feuerriegel, S. 2017. Mycobacterium tuberculosis resistance prediction and lineage classification from genome sequencing: comparison of automated analysis tools. Scientific Reports 7: 46327 DOI: 10.1038/srep46327.

Stajich, J. E., Block, D., Boulez, K., Brenner, S. E., Chervitz, S. A., Dagdigian, C., Fuellen, G., Gilbert, J. G., Korf, I., Lapp, H., Lehvaslaiho, H., Matsalla, C., Mungall, C. J., Osborne, B. I., Pocock, M. R., Schattner, P., Senger, M., Stein, L. D., Stupka, E., Wilkinson, M. D. and Birney, E. 2002. The Bioperl toolkit: Perl modules for the life sciences. Genome Research 12: 1611-1618 DOI: 10.1101/gr.361602. 
395

396

397

398

399

400

401

402

403

404

405

406
Steiner, A., Stucki, D., Coscolla, M., Borrell, S. and Gagneux, S. 2014. KvarQ: targeted and direct variant calling from fastq reads of bacterial genomes. BMC Genomics 15: 881 DOI: 10.1186/1471-2164-15-881.

Walker, T. M., Cruz, A. L. G., Peto, T. E., Smith, E. G., Esmail, H. and Crook, D. W. 2017. Tuberculosis is changing. The Lancet Infectious Diseases 17: 359-361 DOI: 10.1016/s1473-3099(17)30123-8.

Walker, T. M., Ip, C. L., Harrell, R. H., Evans, J. T., Kapatai, G., Dedicoat, M. J., Eyre, D. W., Wilson, D. J., Hawkey, P. M., Crook, D. W., Parkhill, J., Harris, D., Walker, A. S., Bowden, R., Monk, P., Smith, E. G. and Peto, T. E. 2013. Whole-genome sequencing to delineate Mycobacterium tuberculosis outbreaks: a retrospective observational study. The Lancet Infectious Diseases 13: 137-146 DOI: 10.1016/S14733099(12)70277-3.

Walker, T. M., Merker, M., Knoblauch, A. M., Helbling, P., Schoch, O. D., van der Werf, M. J., Kranzer, K., Fiebig, L., Kroger, S., Haas, W., Hoffmann, H., Indra, A., Egli, A., Cirillo, D. M., Robert, J., Rogers, T. R., Groenheit, R., Mengshoel, A. T., Mathys, V., Haanpera, M., Soolingen, D. V., Niemann, S., Bottger, E. C., Keller, P. M. and Consortium, M.-T. C. 2018. A cluster of multidrug-resistant Mycobacterium tuberculosis among patients arriving in Europe from the Horn of Africa: a molecular epidemiological study. The Lancet Infectious Diseases 18: 431-440 DOI: 10.1016/S1473-3099(18)30004-5.

World Health Organization. 2017. Global tuberculosis report 2017.

Zignol, M., Cabibbe, A. M., Dean, A. S., Glaziou, P., Alikhanova, N., Ama, C., Andres, S., Barbova, A., Borbe-Reyes, A., Chin, D. P., Cirillo, D. M., Colvin, C., Dadu, A., Dreyer, A., Driesen, M., Gilpin, C., Hasan, R., Hasan, Z., Hoffner, S., Hussain, A., Ismail, N., Kamal, S. M. M., Khanzada, F. M., Kimerling, M., Kohl, T. A., Mansjo, M., Miotto, P., Mukadi, Y. D., Mvusi, L., Niemann, S., Omar, S. V., Rigouts, L., Schito, M., Sela, I., Seyfaddinova, M., Skenders, G., Skrahina, A., Tahseen, S., Wells, W. A., Zhurilo, A., Weyer, K., Floyd, K. and Raviglione, M. C. 2018. Genetic sequencing for surveillance of drug resistance in tuberculosis in highly endemic countries: a multi-country population-based surveillance study. The Lancet Infectious Diseases 18: 675-683 DOI: 10.1016/S1473-3099(18)30073-2. 


\section{Figure 1}

\section{Schematic representation of the MTBseq workflow}

Modules encapsulating specific functionality shown in blue boxes.

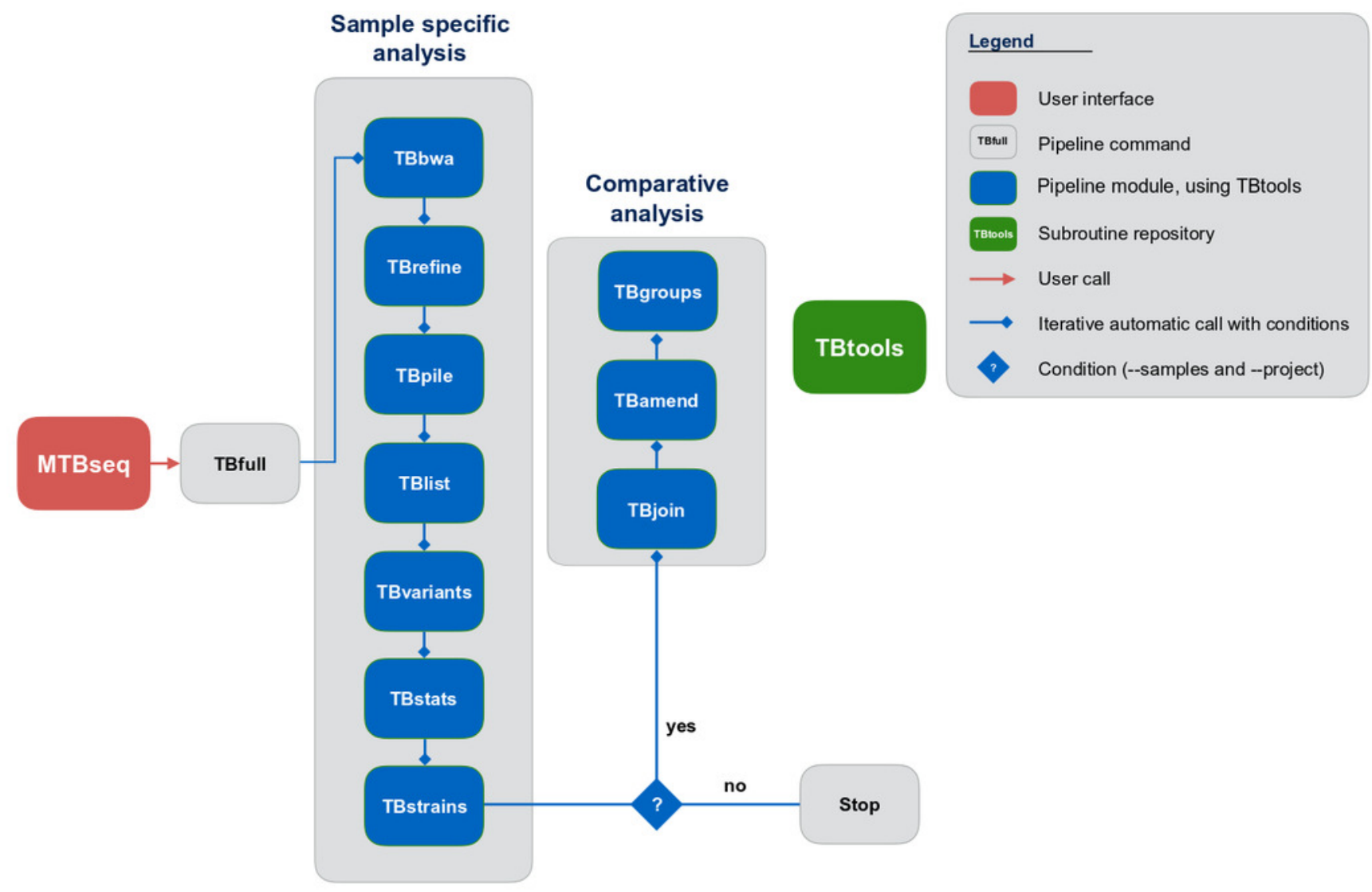




\section{Figure 2}

Maximum likelihood phylogenetic tree.

Maximum likelihood phylogenetic tree constructed from the aligned set of SNP positions determined by MTBseq from a collection of 26 MTBC isolates suspected to form an outbreak ( Kohl et al., 2014 ). For tree construction, we employed the program FastTree version 2 (Price et al., 2010) in the double precision built with a general time reversible (GTR) substitution model, 1,000 resamples, and Gamma20 likelihood optimization. The resulting tree was visualized with the FigTree and EvolView (He et al., 2016) tools. d12_groups: Groups of clustered isolates were determined by MTBseq with a maximum distance threshold of 12 SNPs using single-linkage clustering and the detected groups are indicated by the colored sample labels. Support value: Reliability values for splits based on resampling over $80 \%$ are shown. 


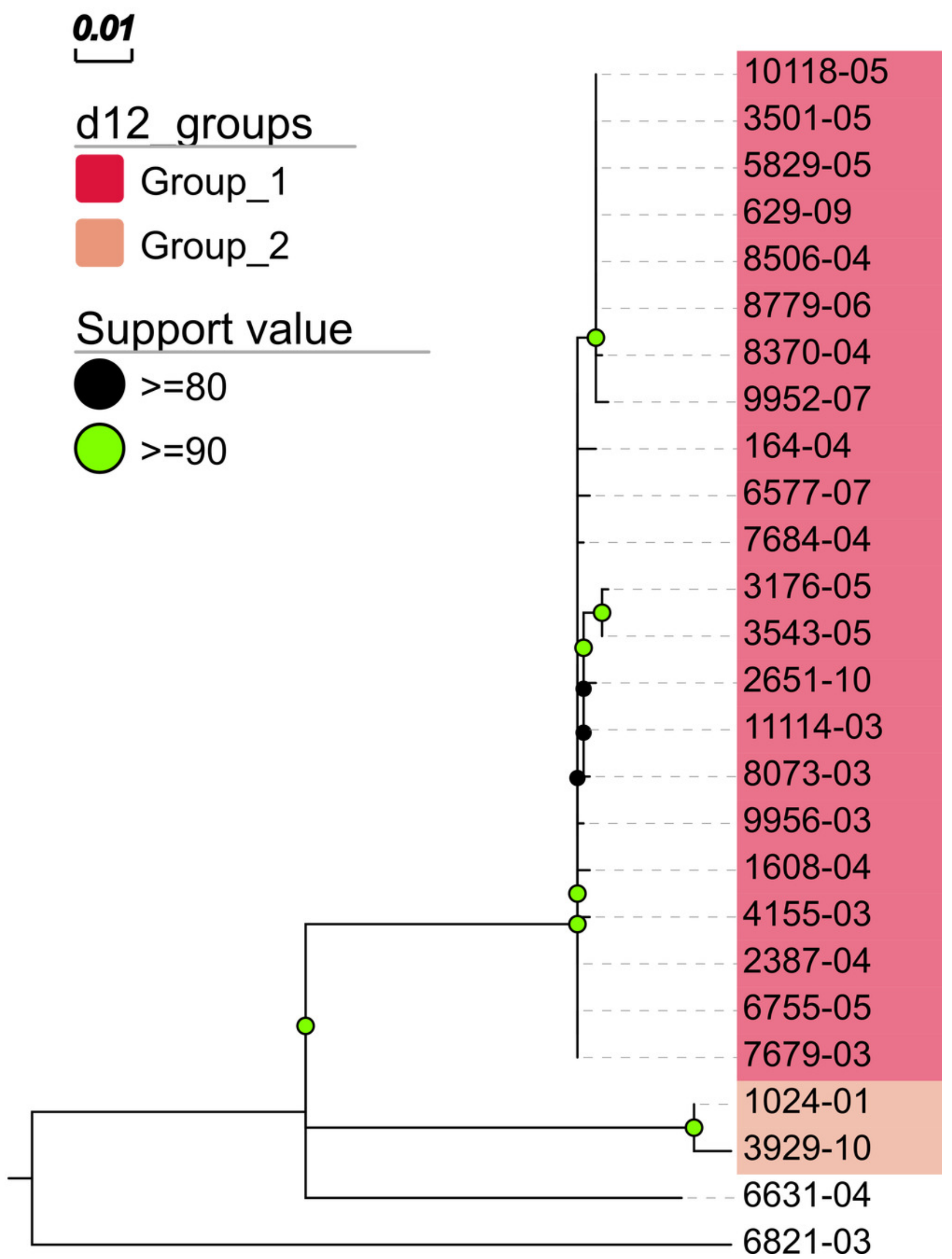




\section{Figure 3}

Pairwise distance matrix

Pairwise distance matrix calculated by MTBseq from a set of 26 MTBC isolates with identical traditional genotyping patterns suspected to form an outbreak (Kohl et al., 2014). The distance between samples is calculated from the detected variants and smaller distances indicate more closely related samples. Out of the 26 isolates, 22 have overall small pairwise distances indicative of a common cluster. The respective entries for the four remaining isolates are marked in blue (1024-01, 3929-10, 6631-04, 6821-03).

\begin{tabular}{|c|c|c|c|c|c|c|c|c|c|c|c|c|c|c|c|c|c|c|c|c|c|c|c|c|c|c|}
\hline $9956-03$ & 7 & 100 & 2 & 3 & 4 & 1 & 4 & 6 & 4 & 5 & 106 & 3 & 4 & 4 & 3 & 101 & 1 & 183 & 1 & 2 & 3 & 5 & 4 & 4 & 6 & NA \\
\hline 9952-07 & 2 & 104 & 6 & 7 & 8 & 5 & 8 & 10 & 2 & 9 & 110 & 7 & 2 & 2 & 7 & 105 & 5 & 187 & 5 & 6 & 7 & 3 & 2 & 2 & NA & 6 \\
\hline 8779-06 & 0 & 102 & 4 & 5 & 6 & 3 & 6 & 8 & 0 & 7 & 108 & 5 & 0 & 0 & 5 & 103 & 3 & 185 & 3 & 4 & 5 & 1 & 0 & NA & 2 & 4 \\
\hline $8506-04$ & 0 & 102 & 4 & 5 & 6 & 3 & 6 & 8 & 0 & 7 & 108 & 5 & 0 & 0 & 5 & 103 & 3 & 185 & 3 & 4 & 5 & 1 & NA & 0 & 2 & 4 \\
\hline $8370-04$ & 1 & 103 & 5 & 6 & 7 & 4 & 7 & 9 & 1 & 8 & 109 & 6 & 1 & 1 & 6 & 104 & 4 & 186 & 4 & 5 & 6 & NA & 1 & 1 & 3 & 5 \\
\hline 8073-03 & 5 & 101 & 1 & 4 & 5 & 2 & 3 & 5 & 5 & 4 & 107 & 4 & 5 & 5 & 4 & 102 & 2 & 184 & 2 & 3 & NA & 6 & 5 & 5 & 7 & 3 \\
\hline 7684-04 & 4 & 100 & 2 & 3 & 4 & 1 & 4 & 6 & 4 & 5 & 106 & 3 & 4 & 4 & 3 & 101 & 1 & 183 & 1 & NA & 3 & 5 & 4 & 4 & 6 & 2 \\
\hline $7679-03$ & 3 & 99 & 1 & 2 & 3 & 0 & 3 & 5 & 3 & 4 & 105 & 2 & 3 & 3 & 2 & 100 & 0 & 182 & NA & 1 & 2 & 4 & 3 & 3 & 5 & 1 \\
\hline $6821-03$ & 185 & 199 & 183 & 184 & 185 & 182 & 185 & 187 & 185 & 186 & 205 & 184 & 185 & 185 & 184 & 186 & 182 & NA & 182 & 183 & 184 & 186 & 185 & 185 & 187 & 183 \\
\hline 6755-05 & 3 & 99 & 1 & 2 & 3 & 0 & 3 & 5 & 3 & 4 & 105 & 2 & 3 & 3 & 2 & 100 & NA & 182 & 0 & 1 & 2 & 4 & 3 & 3 & 5 & 1 \\
\hline $6631-04$ & 103 & 117 & 101 & 102 & 103 & 100 & 103 & 105 & 103 & 104 & 123 & 102 & 103 & 103 & 102 & NA & 100 & 186 & 100 & 101 & 102 & 104 & 103 & 103 & 105 & 101 \\
\hline 6577-07 & 5 & 101 & 3 & 4 & 5 & 2 & 5 & 7 & 5 & 6 & 107 & 4 & 5 & 5 & NA & 102 & 2 & 184 & 2 & 3 & 4 & 6 & 5 & 5 & 7 & 3 \\
\hline $629-09$ & 0 & 102 & 4 & 5 & 6 & 3 & 6 & 8 & 0 & 7 & 108 & 5 & 0 & NA & 5 & 103 & 3 & 185 & 3 & 4 & 5 & 1 & 0 & 0 & 2 & 4 \\
\hline 5829-05 & 0 & 102 & 4 & 5 & 6 & 3 & 6 & 8 & 0 & 7 & 108 & 5 & NA & 0 & 5 & 103 & 3 & 185 & 3 & 4 & 5 & 1 & 0 & 0 & 2 & 4 \\
\hline 4155-03 & 5 & 101 & 3 & 4 & 5 & 2 & 5 & 7 & 5 & 6 & 107 & NA & 5 & 5 & 4 & 102 & 2 & 184 & 2 & 3 & 4 & 6 & 5 & 5 & 7 & 3 \\
\hline $3929-10$ & 108 & 6 & 106 & 107 & 108 & 105 & 108 & 110 & 108 & 109 & NA & 107 & 108 & 108 & 107 & 123 & 105 & 205 & 105 & 106 & 107 & 109 & 108 & 108 & 110 & 106 \\
\hline $3543-05$ & -7 & 103 & 3 & 6 & 7 & 4 & 5 & 1 & 7 & NA & 109 & 6 & 7 & 7 & 6 & 104 & 4 & 186 & 4 & 5 & 4 & 8 & 7 & 7 & 9 & 5 \\
\hline $3501-05$ & - & 102 & 4 & 5 & 6 & 3 & 6 & 8 & NA & 7 & 108 & 5 & 0 & 0 & 5 & 103 & 3 & 185 & 3 & 4 & 5 & 1 & 0 & 0 & 2 & 4 \\
\hline $3176-05$ & 8 & 104 & 4 & 7 & 8 & 5 & 6 & NA & 8 & 1 & 110 & 7 & 8 & 8 & 7 & 105 & 5 & 187 & 5 & 6 & 5 & 9 & 8 & 8 & 10 & 6 \\
\hline $2651-10$ & 6 & 102 & 2 & 5 & 6 & 3 & NA & 6 & 6 & 5 & 108 & 5 & 6 & 6 & 5 & 103 & 3 & 185 & 3 & 4 & 3 & 7 & 6 & 6 & 8 & 4 \\
\hline 2387-04 & 3 & 99 & 1 & 2 & 3 & NA & 3 & 5 & 3 & 4 & 105 & 2 & 3 & 3 & 2 & 100 & 0 & 182 & 0 & 1 & 2 & 4 & 3 & 3 & 5 & 1 \\
\hline 164-04 & 6 & 102 & 4 & 5 & NA & 3 & 6 & 8 & 6 & 7 & 108 & 5 & 6 & 6 & 5 & 103 & 3 & 185 & 3 & 4 & 5 & 7 & 6 & 6 & 8 & 4 \\
\hline 1608-04 & - & 101 & 3 & NA & 5 & 2 & 5 & 7 & 5 & 6 & 107 & 4 & 5 & 5 & 4 & 102 & 2 & 184 & 2 & 3 & 4 & 6 & 5 & 5 & 7 & 3 \\
\hline 11114-03 & 4 & 100 & NA & 3 & 4 & 1 & 2 & 4 & 4 & 3 & 106 & 3 & 4 & 4 & 3 & 101 & 1 & 183 & 1 & 2 & 1 & 5 & 4 & 4 & 6 & 2 \\
\hline 1024-01 & -102 & NA & 100 & 101 & 102 & 99 & 102 & 104 & 102 & 103 & 6 & 101 & 102 & 102 & 101 & 117 & 99 & 199 & 99 & 100 & 101 & 103 & 102 & 102 & 104 & 100 \\
\hline \multirow[t]{3}{*}{ 10118-05 } & NA & 102 & 4 & 5 & 6 & 3 & 6 & 8 & 0 & 7 & 108 & 5 & 0 & 0 & 5 & 103 & 3 & 185 & 3 & 4 & 5 & 1 & 0 & 0 & 2 & 4 \\
\hline & & 1 & $T$ & 1 & 1 & $T$ & $T$ & $T$ & $T$ & 1 & 1 & 1 & 1 & 1 & 1 & 1 & $T$ & & 1 & $T$ & 1 & $T$ & $T$ & 1 & 1 & 7 \\
\hline & 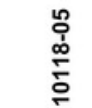 & 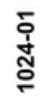 & 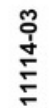 & $\begin{array}{l}\text { ષे } \\
\text { ळ̊ } \\
\stackrel{0}{0}\end{array}$ & 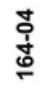 & $\begin{array}{l}\stackrel{+}{+} \\
\stackrel{1}{\infty} \\
\stackrel{\sim}{N}\end{array}$ & $\frac{\text { 움 }}{\text { 융 }}$ & 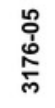 & 눙 & $\begin{array}{l}\text { ஸे } \\
\text { ஸे } \\
\text { ஸ్ల }\end{array}$ & 웋 & 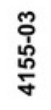 & 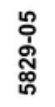 & 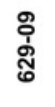 & 容 & 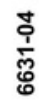 & 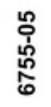 & 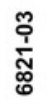 & 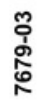 & 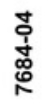 & 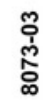 & 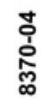 & $\begin{array}{l}\text { 广 } \\
\stackrel{1}{1} \\
\stackrel{0}{0} \\
\infty\end{array}$ & $\begin{array}{l}\stackrel{8}{\circ} \\
\text { ò } \\
\stackrel{\infty}{\$}\end{array}$ & $\begin{array}{l}\text { 엄 } \\
\text { ஸू. } \\
\text { ᄋ̆ }\end{array}$ & $\begin{array}{l}\text { \% } \\
\text { ஸे } \\
\text { ஸू } \\
\text { oे }\end{array}$ \\
\hline
\end{tabular}




\section{Table $\mathbf{1}$ (on next page)}

Sensitivity and specificity for resistance prediction of different tools.

Evaluation of resistance deduction from whole genome sequence data by programs CASTB, Phyresse, kvarQ, MykrobePredictor TB, TBProfiler and MTBseq, with sensitivity (Sens) and specificity (Spec) estimated with 95\% confidence intervals compared to Sanger sequencing results (\#R resistant, \#S sensitive). INH: isoniazid, RMP: rifampicin, SM: streptomycin, EMB: ethambutol, PZA: pyrazinamide. 


\section{Table 1:}

\begin{tabular}{|l|l|l|l|l|l|l|l|l|l|l|l|l|l|}
\hline Antibiotic & \multicolumn{3}{|l|}{ Sanger } & CASTB & PhyResSE & \multicolumn{2}{l|}{ kvarQ } & \multicolumn{2}{l|}{ Mykrobe Predictor TB } & \multicolumn{2}{l|}{ TBProfiler } \\
& \#R & $\# \mathbf{S}$ & Sens & Spec & Sens & Spec & Sens & Spec & Sens & Spec & Sens & Spec \\
\hline INH & 28 & 63 & $89(72,98)$ & $100(92,100)$ & $100(82,100)$ & $98(91,100)$ & $86(67,96)$ & $100(92,100)$ & $89(72,98)$ & $100(92,100)$ & $93(76,99)$ & $84(73,92)$ & $100(82,100)$ \\
RMP & 18 & 73 & $94(73,100)$ & $100(93,100)$ & $100(74,100)$ & $99(93,100)$ & $94(73,100)$ & $100(93,100)$ & $100(74,100)$ & $99(93,100)$ & $100(74,100)$ & $99(93,100)$ & $100(74,100)$ \\
SM & 37 & 54 & $30(16,47)$ & $100(90,100)$ & $100(86,100)$ & $100(90,100)$ & $57(39,73)$ & $100(90,100)$ & $57(39,73)$ & $100(90,100)$ & $57(39,73)$ & $100(90,100)$ & $100(86,100)$ \\
EMB & 15 & 76 & $53(27,79)$ & $100(93,100)$ & $94(70,100)$ & $100(93,100)$ & $53(27,79)$ & $100(93,100)$ & $47(21,73)$ & $99(93,100)$ & $94(70,100)$ & $99(93,100)$ & $100(71,100)$ \\
PZA & 11 & 80 & $45(17,77)$ & $100(93,100)$ & $100(62,100)$ & $99(93,100)$ & $45(17,77)$ & $100(93,100)$ & n.a. & n.a. & $100(62,100)$ & $99(93,100)$ & $100(62,100)$ \\
\hline
\end{tabular}

3

4

5

6

7 